\title{
The Study of Modified Semi-Empirical Mass Formula (SEMF) by Considering Isospin Effects in Liquid Drop Model
}

\author{
Sinta Ayu Sakinah ${ }^{1}$, Eko Tri Sulistyani ${ }^{2}$ \\ ${ }^{1}$ Physics Department, ${ }^{2}$ Research Group of Astrophysics, Cosmology and Mathematical Physics, Faculty of Mathematics and Natural Science \\ Gadjah Mada University, Bulaksumur, Caturtunggal, Yogyakarta 55281, Indonesia. Tel. +62 (274) 6492599, Fax. +62 (274) 565223 \\ Email: sinta.ayu.s@mail.ugm.ac.id ${ }^{1}$, sulistyani@ugm.ac.id ${ }^{2}$
}

\begin{abstract}
We do theoretically study of Modified Semi-Empirical Mass Formula (SEMF) based on macroscopic approach in liquid drop model by considering isospin effects. Isospin is one of internal symmetry properties in hadron group, particularly the nucleon multiplet, it represented by $S U(2)$ isospin group. Hadron is a group of elementary particles take place in the strong interaction. The role of strong interactions represents homogeneous nuclear force, interactions between proton-proton $\left(F_{p p}\right)$, proton-neutron $\left(F_{p n}\right)$, and neutron-neutron $\left(F_{n n}\right)$ are same. In other words, protons and neutrons are indistinguishable because mass (energy) between protons and neutrons is almost the same, by removing charge between them (charge independent). The dependence of isospin effects on nuclear symmetry term and oddeven (pairing) term made the formulation of SEMF should be modificated, in order to obtain nuclear mass and binding energy of a nucleus close to the experimental results. We do two accuracy testing. First, by comparing $\left|\mathrm{M}_{\mathrm{exp}}-\mathrm{M}_{\mathrm{th}}\right|$ for nuclei $\mathrm{Pb} b_{82}^{208}$ using SEMF before and after being modified, the result shows that using SEMF before modification the value of $\left|M_{\exp }-M_{\text {th }}\right| \approx 0,0204 u$ and for modified SEMF we obtained $\left|\mathrm{M}_{\mathrm{exp}}-\mathrm{M}_{\mathrm{th}}\right| \approx 0,0203 u$ at $k=12$. The value of $\left|\mathrm{M}_{\mathrm{exp}}-\mathrm{M}_{\mathrm{th}}\right|$ for modified SEMF is smaller than before modification, it indicates that Modified SEMF is a good formula to calculate the mass of nuclei. Second, by comparing Modified SEMF with other models such as FRDM, HFB-14, and HFB-17 using accuracy parameter in the form of rms deviation $(\sigma(M))$ and number of model parameters $\left(\mathrm{N}_{\mathrm{p}}\right)$. The results show that rms deviation $\left(\sigma(\mathrm{M})\right.$ ) decrease $21 \%$ to 0,516 and number of model parameters $\left(\mathrm{N}_{\mathrm{p}}\right)$ decrease to 15 , consists of 13 macroscopic model parameters $\left(a_{v}, a_{s}, a_{c}, c_{s y m}, \mathcal{K}, a_{\text {pair, }} g_{1}, g_{2}, c_{1}, V_{0}, r_{0}, a, \lambda_{0}\right)$ and two microscopic model parameters $(\gamma$ and $p)$. The value of model parameters was obtained by fitting to experimental results, as a reason it is called semiempiric.
\end{abstract}

Keywords: Isospin effects, Modified Semi-Empirical Mass Formula (SEMF)

Abbreviations: Semi-Empirical Mass Formula (SEMF), Liquid Drop Model (LDM), Finite Range Droplet Model (FRDM), Hartree Fock Bougolibouv (HFB), Atomic Mass Evaluation (AME), Special Unitary Group matrix $2 \times 2(S U(2))$

\section{INTRODUCTION}

Began with the most fundamental question "What is matter made of?". Demoscritus (450 BC) first proposed that everything in the world was made up of tiny particles, it is indivisible and be the root of basic structure of all matter, it called Atom. Atomic research was continued to the end of classical period (18971932). It was known that atom composed of protons and neutrons in nucleus, and electrons orbit around the atomic nucleus (Griffiths D, 2008). Therefore, the mass calculation of atomic nucleus based on number of protons and neutrons.

The theoretical development on calculating the mass of an atomic nucleus attracts more attention when scientists were surprised with the fact that the mass of an atomic nucleus is less than the sum of the individual masses of the free constituent protons and neutrons. The difference between the actual nuclear mass and the mass of all the individual nucleons is called the nuclear binding energy $E_{L D}(A, Z)$. It represents the work necessary to dissociate the nucleus into separate nucleons or conversely, the energy which would be released if the separated nucleons were assembled into a nucleus. The formulation of nuclear binding energy has been developing, one of the most familiar is SemiEmpirical Mass Fomula (SEMF) as the form of macroscopic approach based on Liquid Drop Model (LDM).

Semi-Empirical Mass Formula (SEMF) was first formulated in 1935 by German Physicist C.F Von Weizsäcker, it became the starting point to determine the mass of nuclei, Then in 1936 Bethe tried to improve the SEMF by correcting some coefficients and got better results than before. So it is known as BetheWeizsäcker Semi-Empirical Mass Formula. Furthermore, Strutinsky modified SEMF by adding the constribution of microscopic aspect known as Strutinsky shell correction $\Delta E$.

Previous modifications have been made, but the obtained nuclear mass is not close enough to the experimental result which is comprehensively listed in the database on Atomic Mass Evaluation (AME). It indicated by the large number of rms deviation $(\sigma(M))$ and number of model parameters $\left(N_{p}\right)$. Therefore, SEMF must be modified by taking isospin into account. Isospin roles among hadron, strong interaction particles, particularly the nucleon multiplet. 
Homogeneous nuclear force is manifestation of strong interaction that represented by $S U(2)$ group, it means that interactions between proton-proton $\left(F_{p p}\right)$, protonneutron $\left(F_{p n}\right)$, and neutron-neutron $\left(F_{n n}\right)$ are indistinguishable because mass (energy) between protons and neutrons is almost the same, by removing charge between them (charge independent).

In this paper, we will do theoretically study on how isospin effects nuclear symmetry term and odd-even (pairing) term of Modified Semi-Empirical Mass Formula (SEMF) in Liquid Drop Model (LDM), so the aim to obtain accurate nuclear mass can be reached. Then, We do two accuracy testing. First, by comparing $\left|\mathrm{M}_{\text {exp }}-\mathrm{M}_{\mathrm{th}}\right|$ for nuclei $\mathrm{Pb}_{82}^{208}$ using SEMF before and after being modified. Second, compare Modified SEMF with other models such as FRDM, HFB-14, and HFB17 using accuracy parameter in the form of $\mathrm{rms}$ deviation $\left(\sigma(M)=\left[\frac{1}{n} \sum_{i=1}^{n}\left(M_{\text {exp }}^{i}-M_{t h}^{i}\right)^{2}\right]^{1 / 2}\right)$ and number of model parameters $\left(\mathrm{N}_{\mathrm{p}}\right)$ used in each models.

\section{Liquid Drop Model}

Liquid Drop Model (LDM) was proposed by Niels Bohr in 1937, it suggest that the nucleus is analogous like the molecules in a drop of liquid. This analogy based on some assumptions that lies on some identical characteristic of nuclei and liquid droplets, as follows:

1. The drop of liquid is spherical because of the symmetrical surface tension forces which act towards the center. The nucleus is assumed to be spherical

2. The molecules in a liquid drop interact over short ranges compared with the diameter of drop, like nucleons in the nucleus

3. The molecules move short distance in thermal velocities, if the thermal agiation is increased by raising the temperature, evaporation of molecule takes place. The nucleons in a nucleus also have kinetic energy, if energy is given to the nucleus by a bombarding particles, a compound nucleus is formed which emits nucleons almost immediately

4. The compound nucleus or liquid drop may get deexcited by undergoing any of following changes (listed in table 1)

Table 1. Liquid droplets analogous with nuclei on deexcited energy process.

\begin{tabular}{ll}
\hline Liquid Droplets & Nuclei \\
\hline Releasing heat & Emission of radiation \\
Evaporation some particles & Emission of nucleon \\
Breaking up into drop & Nuclear Fission \\
\hline
\end{tabular}

Not only from assumptions above, there are some essential assumptions (Meyerhof WE, 1967):

1. The nucleus consists of incompressible matter so that $\mathrm{R} \sim A^{1 / 3}$
2. The nuclear force is identical for every nucleon and in particular does not depend on whether it is a neutron or a proton

3. The nuclear force saturates

Those assumptions lead to formulate the SemiEmpirical Mass Formula (SEMF) on macroscopic aspect of Liquid Drop Model (LDM). Macroscopic aspect means protons and neutrons in nucleus are not assumed separately, but in unity of one family, nucleon. Entire nucleon in nucleus is assumed homogen.

\section{Semi-Empirical Mass Formula (SEMF)}

Semi-Empirical Mass Formula (SEMF) that formulated in 1935 by C.F Von Weizsäcker based on analogy from Liquid Drop Model (LDM) was explained before. It constributed on macroscopic aspect such as Volume effect, Surface effect, Coulomb effect, Symmetry effect, and Odd-Even (Pairing) effect.

\section{Volume Effect}

The nuclear binding energy affects both protons and neutrons or depends pincipally on the mass number $A$ which means that it is proportional to the volume of nucleus. The equation is written as follows:

$$
E_{v}=a_{v} A,
$$

where $E_{v}$ is volume energy, $a_{v}$ is volume coefficient, and $A$ is the atomic mass of nucleus, $A$ also known as sum of protons and neutons in a nucleus it is written by $A=Z+N$.

\section{Surface Effect}

Surface Area of a nucleus is assumed as surface area of a sphere analogous from the assumption of LDM. Form the equation below:

$$
4 \pi R^{2}=4 \pi R_{0}^{2} A^{2 / 3} .
$$

with radius of a sphere is

$$
R=R_{0} A^{\frac{1}{3}}
$$

where $R_{0}$ is called radius constant and has the values $1,2 F$ for electron scattering on nuclei, and $1,4 F$ for nuclear particle scattering on nuclei. Surface area of nucleus proportional to $R^{2}$. Surface energy of nucleus proportional to surface area so it is proportional to the following equation:

$$
R^{2} \sim A^{2 / 3}=a_{s} A^{2 / 3}
$$

Surface energy can be written by

$$
E_{s}=-a_{s} A^{2 / 3} \text {, }
$$

where $E_{s}$ is surface energy of nucleus, $a_{s}$ is surface coefficient, and $A$ is the atomic mass of nucleus. The negative value of $E_{S}$ due to the nuclear force for the surface-nucleons is weaker than nucleons that locate inside the nucleus. 


\section{Coulomb Effect}

Coulomb forces act between pairs of protons, affect on electrostatic repulsion between protons. This repulsions have opposite direction of nuclear binding energy. Therefore, Coulomb energy will reduce the total nuclear binding energy in the form:

$$
E_{c}=-a_{c} \frac{Z(Z-1)}{A^{1 / 3}}
$$

where $E_{c}$ is Coulomb energy of nucleus, $a_{c}$ is Coulomb coefficient, $Z$ is number of proton, and $A$ is the atomic mass of nucleus.

\section{Symmetry Effect}

Nuclear symmetry effect is influenced by the difference number of protons $Z$ and neutrons $N$ in nucleus. The difference number of neutrons and protons $(N-Z)$ can reduce the stability of nucleus and decrease the total nuclear binding energy, this constribute to the negative value of asymmetry energy in the form:

$$
E_{a}=-a_{a} \frac{(N-Z)^{2}}{A} .
$$

where $E_{a}$ is asymmetry energy of nucleus, $a_{a}$ is asymmetry coefficient, $N$ is number of neutrons, $Z$ is number of protons and $A$ is the atomic mass of nucleus.

\section{Odd-Even (Pairing) Effect}

Other factor that impact the calculation of nuclear binding energy is the number of protons $(Z)$ and neutrons $(N)$ which is odd or even number. This effect arises from the tendency of proton pairs and neutron pairs. Even-even nuclei is the most stable and hence have higher binding energy than would otherwise be expected. In contrast, odd-odd nuclei which mean number of protons are odd, and number of neutrons are odd have both unpaired protons and neutrons and have relatively low binding energy. The pairing term correction is positive for even-even nuclei (increase the binding energy), zero for odd-even and even-odd nuclei (no constribution), and negative for odd-odd nuclei (decrease the binding energy), it can be written as follows

ä $(A, Z)\left\{\begin{array}{cc}+a_{p} A^{-3 / 4} & \text { for } Z \text { (even), } N \text { (even) } \\ 0 & \text { for } A \text { odd } \\ -a_{p} A^{-3 / 4} & \text { for } Z(\text { odd }), N(\text { odd })\end{array}\right.$

For simplification, pairing energy is

$$
\ddot{\mathrm{a}}(A, Z)=E_{p}=( \pm, 0) \frac{a_{p}}{A^{3 / 4}}
$$

where $E_{p}$ is pairing energy of nucleus, $a_{p}$ is asymmetry coefficient, and $A$ is the atomic mass of nucleus.

Hence, the final expression for the nuclear binding energy of Bethe-Weizsäcker Semi-Empirical Mass Formula (SEMF) is

$E_{L D}(A, Z)=a_{V} A-a_{S} A^{2 / 3}-a_{c} \frac{Z(Z-1)}{A^{\frac{1}{3}}}-$

$a_{a} \frac{(N-Z)^{2}}{A}( \pm, 0) \frac{a_{p}}{A^{3 / 4}}$.
The value of those coefficients that give a good fit with the data are as follows:

$a_{V}=14 \mathrm{Mev} ; a_{s}=13 \mathrm{Mev} ; a_{c}=0,6 \mathrm{Mev} ; a_{a}=$ $19 \mathrm{Mev} ; a_{p}=34 \mathrm{Mev}$

If $1 u=931,5 \mathrm{Mev} / \mathrm{c}^{2}$, Hence

$a_{V}=0,0169123 u ; a_{s}=0,0019114 u ; a_{c}=$

$0,0007626 u ; \quad a_{a}=0,10175 u ; a_{p}=0,036 u$

A set of coefficients $a_{V}, a_{s}, a_{c}, a_{a}$, and $a_{p}$ were obtained by fitting with the empirical results, as the reason it is called semi-empiric.

\section{Isospin on Nucleon}

Heisenberg (1932) proposed that if the electric charge of proton "switch off" there would be no way to distinguish between a proton and neutron. This idea arises due to proton and neutron have similar masses with respect to the strong interaction (table 2). Hence, the nuclear force is identic $\left(F_{p p} \approx F_{p n} \approx F_{n n}\right)$ and it found to be approximately charge independent. Furthermore, neuton and proton should be considered as two states of a single entity, the nucleon. This characteristic is called isospin on nucleon.

Table 2. Properties and Mass of protons and neutrons (Greiner W and Müller B, 1989).

\begin{tabular}{llll}
\hline Nucleon & Mass (Mev) & Mass difference & Spin \\
\hline proton & 983,213 & 1,294 & $1 / 2$ \\
neutron & 939,507 & & $1 / 2$ \\
\hline
\end{tabular}

Isospin is one of the symmetry internal that occurs on nucleon. Symmetries are conveniently described by a group theory (Greiner W and Müller B, 1989) and Isospin is represented by Special Unitary Group $S U(2)$. It called Unitary matrix when fulfilled the relation

$$
\widehat{U} \widehat{U}^{\dagger}=\widehat{U}^{\dagger} \widehat{U}=I,
$$

And it called special if

$$
\operatorname{det} \widehat{U}=1 .
$$

Operator $\widehat{U}$ is the matrix $2 \times 2$, with the form (Greiner W and Müller B, 1989)

$\widehat{U}_{I S}(\stackrel{a}{\mathrm{a}})=\left(\begin{array}{cc}\cos \left(\frac{\AA}{2}\right)-i n_{3} \sin \left(\frac{a}{2}\right) & -\left(n_{2}+i n_{1}\right) \sin \left(\frac{\AA}{2}\right) \\ \left(n_{2}-i n_{1}\right) \sin \left(\frac{a}{2}\right) & \cos \left(\frac{a}{2}\right)+i n_{3} \sin \left(\frac{a}{2}\right)\end{array}\right)$

where $\mathrm{a}$ is the angles

$\stackrel{\circ}{\mathrm{a}}=\left\{\stackrel{\circ}{1}_{1}, \stackrel{\circ}{2}_{2}, \stackrel{\circ}{a}_{3}\right\}=\stackrel{\circ}{\mathrm{a}}\left\{n_{1}+n_{2}+n_{3}\right\}=\stackrel{\circ}{\mathrm{a}} n_{\mathrm{o}^{\prime}}$

Characterize rotations about three axes of the abstract iso-space which can also be interpreted as rotation through å about this axis in the $n_{0}$ directions. The proof that isospin operators $\widehat{U}_{I S}$ are clearly unitary from the equation (13). 




Then it is satisfied the equation (14) by taking the determinant of the matrix $2 \times 2$ from the equation (15), we obtained

$$
\operatorname{det} \widehat{U}_{I S}(\mathrm{a})=\cos ^{2}\left(\frac{\AA}{2}\right)+n_{3}^{2} \sin ^{2}\left(\frac{\AA}{2}\right)+\left(n_{2}^{2}+n_{1}^{2}\right) \sin ^{2}\left(\frac{\AA}{2}\right)=\cos ^{2}\left(\frac{\AA}{2}\right)+\sin ^{2}\left(\frac{\AA}{2}\right)=1
$$

Hence, it proved that isospin operator $\widehat{U}_{I S}$ can be represented by Special Unitary Group $S U(2)$ which is in the same group of strong interaction that act on nucleon. Now, the proof that nuclear forces as the manifestation of strong interaction are identic $\left(F_{p p} \approx\right.$ $F_{p n} \approx F_{n n}$ ) or charge independence of nuclear forces as a consequence of the isospin invariance of the strong interaction as follows (Greiner W and Müller B 1989):

$$
\left[\widehat{H}_{\text {strong }}, \widehat{U}_{I S}(\mathrm{a})\right]=0
$$

Isospin operator $\widehat{U}_{I S}$ analogous to the rotation operator, so å analogous to the angles, given the equation form of isospin operator $\widehat{U}_{I S}(ð)=e^{-i(ð) \hat{T}_{2}}$. The eigenstate of nucleons can be formed as dirac notation $\left|T, T_{3}\right\rangle$, for proton $|p\rangle=\left|\frac{1}{2}, \frac{1}{2}\right\rangle$ and for neutron $|n\rangle=\left|\frac{1}{2},-\frac{1}{2}\right\rangle$, so the relation for proton satisfied in the form

$$
\begin{aligned}
\text { proton } \rightarrow \widehat{U}_{I S}(ð)\left|\frac{1}{2}, \frac{1}{2}\right\rangle & =e^{-i(ð) \hat{T}_{2}}\left|\frac{1}{2}, \frac{1}{2}\right\rangle=e^{-i(ð / 2) \hat{\hat{o}}_{2}}\left|\frac{1}{2}, \frac{1}{2}\right\rangle \\
& =\left(\cos \frac{1}{2} ð-i \hat{\hat{O}}_{2} \sin \frac{1}{2} \text { ð) }\left|\frac{1}{2}, \frac{1}{2}\right\rangle=-i\left(\begin{array}{cc}
0 & -i \\
i & 0
\end{array}\right)\left(\begin{array}{l}
1 \\
0
\end{array}\right)=\left(\begin{array}{cc}
0 & -1 \\
1 & 0
\end{array}\right)\left(\begin{array}{l}
1 \\
0
\end{array}\right)=\left(\begin{array}{l}
0 \\
1
\end{array}\right)=\left|\frac{1}{2},-\frac{1}{2}\right\rangle\right.
\end{aligned}
$$

So, $e^{-i(ð) \hat{T}_{2}}|p\rangle=|n\rangle$, and for neutron is

$$
\begin{aligned}
\text { neutron } \rightarrow \widehat{U}_{I S}(ð)\left|\frac{1}{2},-\frac{1}{2}\right\rangle & =e^{-i(ð) \hat{T}_{2}}\left|\frac{1}{2},-\frac{1}{2}\right\rangle=e^{-i(ð / 2) \hat{\hat{o}}_{2}}\left|\frac{1}{2},-\frac{1}{2}\right\rangle=-i\left(\begin{array}{cc}
0 & -i \\
i & 0
\end{array}\right)\left(\begin{array}{l}
0 \\
1
\end{array}\right)=\left(\begin{array}{cc}
0 & -1 \\
1 & 0
\end{array}\right)\left(\begin{array}{l}
0 \\
1
\end{array}\right) \\
& =-\left(\begin{array}{l}
1 \\
0
\end{array}\right)=-\left|\frac{1}{2}, \frac{1}{2}\right\rangle
\end{aligned}
$$

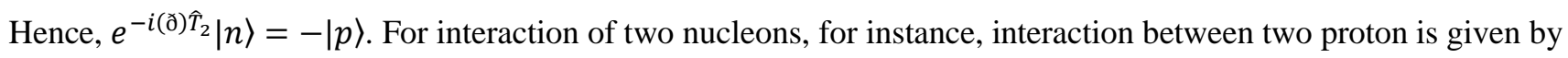

$$
\begin{aligned}
\left\langle p(1) p(2)\left|\widehat{H}_{\text {strong }}\right| p(1) p(2)\right\rangle & =\left\langle e^{-i ð\left(\widehat{T}_{2}(1)+\widehat{T}_{2}(2)\right)}|n(1) n(2)| \widehat{H}_{\text {strong }} \times e^{-i ð\left(\widehat{T}_{2}(1)+\widehat{T}_{2}(2)\right)} \mid n(1) n(2)\right\rangle \\
& =\left\langle n(1) n(2)\left|e^{+i ð\left(\widehat{T}_{2}(1)+\widehat{T}_{2}(2)\right)}\right| \widehat{H}_{\text {strong }} \times e^{-i ð\left(\widehat{T}_{2}(1)+\widehat{T}_{2}(2)\right)} \mid n(1) n(2)\right\rangle \\
& =\left\langle n(1) n(2)\left|\widehat{H}_{\text {strong }}\right| n(1) n(2)\right\rangle
\end{aligned}
$$

This form is simplified to be $\left\langle p p\left|\widehat{H}_{\text {strong }}\right| p p\right\rangle=$ $\left\langle n n\left|\widehat{H}_{\text {strong }}\right| n n\right\rangle$. we conclude that the interaction of two protons is equal to the interaction of two neutrons. It means that nuclear forces are identic or homogen, it fits to the second essential assumption of Liquid Drop Model (LDM).

From the general descriptions of isospin above, we have known that homogeneous nuclear forces is the consequence of the isospin invariance of the strong interaction, it represented by $S U(2)$ group, it is also the reason why isospin should take into account for calculating binding energy. Isospin acts on nucleon (proton and neutron), so it affects straighly to nuclear symmetry term and odd-even (pairing) term of Modified Semi-Empirical Mass Formula (SEMF) by formula which has functions number of protons $Z$ and number of neutrons $N$

$$
I(Z, N)=\frac{(N-Z)}{N+Z}
$$


where $A=N+Z$, it simplified to be

$$
I=\frac{(N-Z)}{A} \text {. }
$$

For more details explanation of relation between Isospin effects and the modified SEMF, see the figure 1 below:

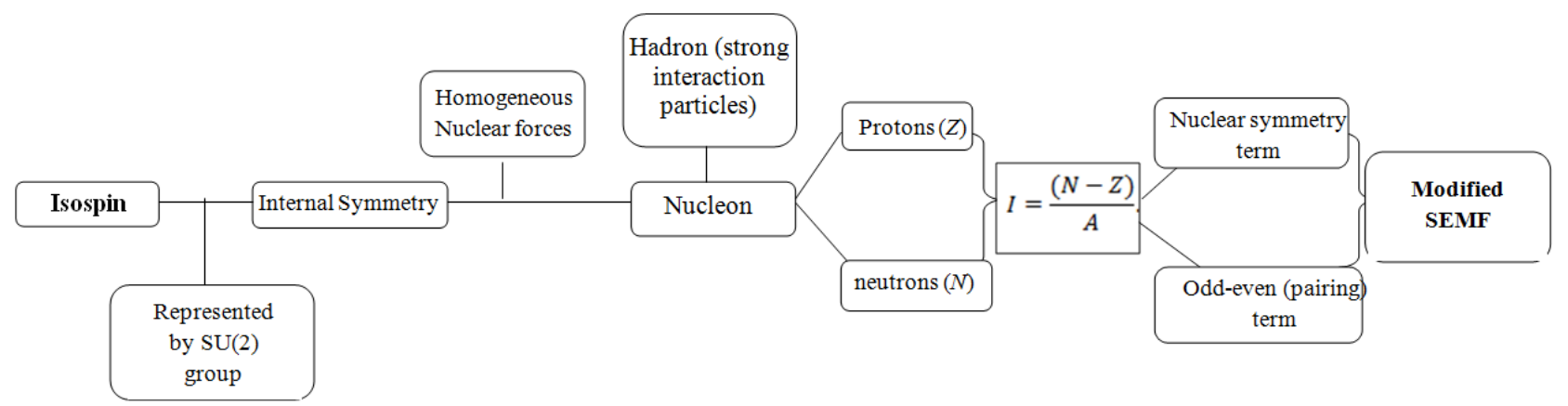

Figure 1. The Relation of Isospin on nucleon to Modified Semi-Empirical Mass Formula (SEMF).

\section{RESULTS AND DISCUSSION}

\section{Results}

In this section, there are two main results. First, after knowing the urgency of isospin takes place on SemiEmpirical Mass Formula (SEMF), then explain how isospin equation subtitutes to make it become Modified Semi-Empirical Mass Formula (SEMF). Second, Test the Modified Semi-Empirical Mass Formula (SEMF), began with comparing SEMF before and after modified for nuclei $\mathrm{Pb}_{82}^{208}$, then by comparing Modified SemiEmpirical Mass Formula (SEMF) based on LDM with others model such as FRDM, HFB-14, and HFB-17 using accuracy parameters in the form of rms deviation (ó(M)) and number of model parameters $\left(\mathrm{N}_{\mathrm{p}}\right)$.

\section{Modified Semi-Empirical Mass Formula (SEMF) by Considering Isospin Effects}

We start with reintroduce the macroscopic part of SEMF from LDM on equation (10) then adding the microscopic part or Strutinsky Shell Correction $(\Delta E)$,

$E_{L D}(A, Z)=a_{V} A-a_{s} A^{\frac{2}{3}}-a_{c} \frac{Z(Z-1)}{A^{\frac{1}{3}}}-a_{a} \frac{(N-Z)^{2}}{A}( \pm, 0) \frac{a_{p}}{A^{3 / 4}}+\Delta E$,

The five terms constribute on macroscopic aspects, and the last term $\Delta E$ constribute the microscopic aspect. The difference between macroscopic and microscopic is the way to look protons and neutrons as entity nucleon which is homogen or not. Therefore, on macroscopic aspects we can assume that the whole nucleons on a nucleus will act the same, then give the average value of macroscopic properties were represented by average binding energy that can be handled by Liquid Drop Model (LDM). In contrast, on microscopic aspect, protons and neutrons are seen separately so more convenient if we use the shell model for fulfilled the equation below:

$$
\ddot{\mathrm{A}} E=c_{1}\left(E_{s h}(Z)+E_{s h}(N)\right),
$$

where $E_{s h}(Z)$ and $E_{s h}(N)$ constribute microscopic energies (shell energies) for protons $(Z)$ and neutrons $(N)$, respectively (Wang $\mathrm{N}$ et al., 2010).

Based on previous discussion that isospin has to take into account of nuclear binding energy, and it leads to Modified SEMF by constribute directly on symmetry term and odd-even (pairing term) which are straighly focus on number of protons $(Z)$ and neutrons $(N)$ in the form $I=\frac{(N-Z)}{A}$. In addition, the influence of nuclear deformation on the macroscopic energy given by (Wang $\mathrm{N}$ et al., 2010).

$$
\prod_{k \geq 2}\left(1+b_{k} \hat{a}_{k}^{2}\right)
$$

It is obtained by using the skyrme energy density functional approach which is $\hat{\mathrm{a}}_{k}=0,01$ and $b_{k}$ is the form of mass dependence of nucleus $(A)$ as follows:

$$
b_{k}=\left(\frac{k}{2}\right) g_{1} A^{1 / 3}+\left(\frac{k}{2}\right) g_{2} A^{-1 / 3},
$$

where $k \geq 2$, and value of model parameters $g_{1}=$ $0,0074, g_{2}=-0,38$ are obtained by fitting the experimental results. It is also needed to substitute in the new form of modified SEMF. Therefore, it satisfied the following equation.

$E(A, Z, \hat{\mathrm{a}})=E_{L D^{\prime}}(A, Z) \prod_{K \geq 2}\left(1+b_{k} \hat{\mathrm{a}}_{k}^{2}+\Delta E(A, Z, \hat{\mathrm{a}})\right.$,

where $E_{L D^{\prime}}(A, Z)$ represents binding energy after modified with considering isospin effect $I$ and the Coulomb exchange correction and surface diffuseness correction to the Coulomb energy is approximately taken into account as the term $Z^{-2 / 3}$ given by: 


$$
\begin{gathered}
E_{L D^{\prime}}(A, Z)=a_{V} A+a_{S} A^{2 / 3}+a_{c} \frac{Z(Z-1)}{A^{1 / 3}}(1- \\
\left.Z^{-2 / 3}\right)+a_{s y m} I^{2} A+a_{\text {pair }} A^{-1 / 3} \ddot{a}_{n p},
\end{gathered}
$$

Isospin affects on nuclear symmetry coefficient $\left(a_{s y m}\right)$ as follows:

$a_{\text {sym }}=c_{\text {sym }}\left[1-\frac{\mathcal{K}}{A^{1 / 3}}+\frac{2-|I|}{2+|I| A}\right]$

The value of model parameters such as $c_{\text {sym }}=$ $29,2876 \mathrm{Mev}$, and $\mathcal{K}=1,4492$ were obtained by fitting the experimental results. Moreover, the pairing term is modified from equation (8) to $\ddot{a}_{n p}=\left\{\begin{aligned} 2-|I|: N \text { and } Z \text { even } \\ |I|: N \text { and } Z \text { odd } \\ 1-|I|: N \text { even, } Z \text { odd, and } N>Z \\ 1-|I|: N \text { odd, } Z \text { even, and } N<Z \\ 1 \quad: N \text { even, } Z \text { odd, and } N<Z \\ 1 \quad: N \text { odd, } Z \text { even, and } N>Z\end{aligned}\right.$

If the equation (31) substitutes into equation (30) and describes isospin in the form $I=\frac{(N-Z)}{A}$, the final expression of Modified Semi-Empirical Mass Formula (SEMF) adopted from equation (29) become

$$
\begin{aligned}
E(A, Z, \hat{\mathrm{a}})= & a_{V} A+a_{s} A^{2 / 3}+a_{c} \frac{Z(Z-1)}{A^{1 / 3}}\left(1-Z^{-2 / 3}\right)+\left(c_{\text {sym }}\left[1-\frac{\mathcal{K}}{A^{1 / 3}}+\frac{2-\left|\frac{(N-Z)}{A}\right|}{2+\left|\frac{(N-Z)}{A}\right| A}\right]\right) \frac{(N-Z)^{2}}{A} \\
& +a_{\text {pair }} A^{-1 / 3} \ddot{\mathrm{a}}_{n p} \prod_{K \geq 2}\left(1+b_{k} \hat{\mathrm{a}}_{k}^{2}\right)+\Delta E(A, Z, \hat{\mathrm{a}})
\end{aligned}
$$

By fitting with the experimental results, a set of each coefficients from equation (33) have the values

$a_{V}=-15,5841 \mathrm{Mev} ; a_{s}=18,2359 \mathrm{Mev} ; a_{c}=$ $0,7173 \mathrm{Mev} ; a_{\text {pair }}=-5,5108 \mathrm{Mev}$

\section{Accuracy Testing of Modified Semi-Empirical Mass Formula (SEMF)}

First, by comparing $\left|\mathrm{M}_{\mathrm{exp}}-\mathrm{M}_{\mathrm{th}}\right|$ for nuclei $\mathrm{Pb}_{82}^{208}$ using SEMF before and after being modified, began with SEMF before modification, Nuclei $\mathrm{Pb}_{82}^{208}$ has a mass number $A=208$, number of protons $Z=82$, and number of neutrons $N=126$, it is classified as eveneven nuclei which have both $Z$ and $N$ even. Based on equation (8) for pairing term, it is satisfied $+a_{p} A^{-3 / 4}$. Therefore, the SEMF before modification become

$$
\begin{array}{r}
E_{L D}(A, Z)=a_{V} A-a_{s} A^{\frac{2}{3}}-a_{c} \frac{Z(Z-1)}{A^{\frac{1}{3}}} \\
-a_{a} \frac{(N-Z)^{2}}{A}+\frac{a_{p}}{A^{3 / 4}}
\end{array}
$$

Substitute all the values given to equation (35). It is obtained $E_{L D}(A, Z) \approx 1606,8 \mathrm{Mev}$, while from experimental results $E_{\exp }(A, Z) \approx 1636,5 \mathrm{Mev}$ (Bartel $\mathrm{J}$ et al., 1982). The difference between them is $29,7 \mathrm{Mev}$ which means the calculation for getting binding energy of a nucleus, this case $\mathrm{Pb}_{82}^{208}$ using SEMF before modification is not good enough. In addition, if we calculate the mass of nuclei $\mathrm{Pb}_{82}^{208}$ using the equation below

$$
M_{X_{p}^{A}}=\left[\left(Z m_{p}+N m_{n}\right)-\frac{E_{L D}}{c^{2}}\right] .
$$

Substitute the value of $E_{L D}(A, Z) \approx 1606,8 \mathrm{Mev}$, and nuclei $\mathrm{Pb}_{82}^{208}$ has a mass number $A=208$, number of protons $Z=82$, and number of neutrons $N=126$. We obtained the mass of nuclei $\mathrm{Pb}_{82}^{208}$ theoretically $M_{P b_{82}^{208}} \approx 207,9970003 u$, while in the experimental result listed in Atomic Mass Evaluation 2012 for nuclei $P b_{82}^{208}$ is $M_{\text {exp }}=207,9766 u$ (Wang $\mathrm{N}$ et al. 2012). The difference between them is $\left|M_{\text {exp }}-M_{t h}\right| \approx$ $0,0204 u$.

Now, calculate the mass of nuclei $\mathrm{Pb}_{82}^{208}$ using Modified SEMF just on macroscopic aspect adopted from equation (33) given by.

$$
\begin{aligned}
E(A, Z, \hat{\mathrm{a}})= & a_{V} A+a_{s} A^{2 / 3}+a_{c} \frac{Z(Z-1)}{A^{1 / 3}}\left(1-Z^{-2 / 3}\right)+\left(c_{s y m}\left[1-\frac{\mathcal{K}}{A^{1 / 3}}+\frac{2-\left|\frac{(N-Z)}{A}\right|}{\left.2+\left|\frac{(N-Z)}{A}\right| A\right]}\right)\right. \\
& \frac{(N-Z)^{2}}{A}+a_{p a i r} A^{-1 / 3} \ddot{\mathrm{a}}_{n p} \prod_{K \geq 2}\left(1+b_{k} \hat{\mathrm{a}}_{k}^{2}\right)
\end{aligned}
$$


Where nuclei $\mathrm{Pb}_{82}^{208}$ satisfied even-even nuclei with $N>Z$, so from equation (32) substitute $\ddot{a}_{n p}=2-|I|=2-$ $\left|\frac{(N-Z)}{A}\right|$.

$$
\begin{gathered}
E(A, Z, \hat{\mathrm{a}})=a_{V} A+a_{S} A^{2 / 3}+a_{c} \frac{Z(Z-1)}{A^{1 / 3}}\left(1-Z^{-2 / 3}\right)+\left(c_{s y m}\left[1-\frac{\mathcal{K}}{A^{1 / 3}}+\frac{2-\left|\frac{(N-Z)}{A}\right|}{2+\left|\frac{(N-Z)}{A}\right| A}\right]\right) \frac{(N-Z)^{2}}{A}+a_{\text {pair }} A^{-1 / 3}(2- \\
\left.\left|\frac{(N-Z)}{A}\right|\right) \prod_{K \geq 2}\left(1+b_{k} \hat{\mathrm{a}}_{k}^{2}\right)
\end{gathered}
$$

Substitute all the value of constanta and model parameters were given in the previous subsection $(\mathrm{A})$ at $k=2$ to equation (38), we obtained $E(A, Z, \hat{a}) \approx$ $1646,1 \mathrm{Mev}$. This value is closer with the experimental result $E_{\text {exp }}(A, Z) \approx 1636,5 \mathrm{Mev}$ rather than energy binding that was obtained using SEMF before modification.

Then, for getting the mass of nuclei $\mathrm{Pb}_{82}^{208}$, substitute the value of $E(A, Z, \hat{\mathrm{a}}) \approx 1646,1 \mathrm{Mev}$ to equation

$$
M_{X_{p}^{A}}=\left[\left(Z m_{p}+N m_{n}\right)-\frac{E(A, Z, \hat{a})}{c^{2}}\right] .
$$

We obtained $M_{P b_{82}^{208}} \approx 207,954756 u$. The difference between theoritical and experimental result for nuclei $\mathrm{Pb}_{82}^{208}$ is $\left|M_{\text {exp }}-M_{t h}\right| \approx 0,0219 u$. This value is at $k=2$, but when we do calculation for $k>2$, with the same procedurs we got (table 3 )

Table 3. The value of $\left|M_{\exp }-M_{t h}\right|$ using Modified SEMF for nuclei $\mathrm{Pb}_{82}^{208}$ at $2 \leq \mathrm{k} \leq 13$.

\begin{tabular}{llll}
\hline $\boldsymbol{k}$ & $\boldsymbol{b}_{\boldsymbol{k}}$ & $\left(\mathbf{1}+\boldsymbol{b}_{\boldsymbol{k}} \hat{\mathrm{a}}_{\boldsymbol{k}}^{\mathbf{2}}\right)$ & $\left|\boldsymbol{M}_{\boldsymbol{e x p}}-\boldsymbol{M}_{\boldsymbol{t h}}\right|(\boldsymbol{u})$ \\
\hline 2 & $-0,0202901644$ & 0,999997971 & 0,0218854 \\
3 & $-0,0785365763$ & 0,999992146 & 0,0218716 \\
4 & $-0,1688505412$ & 0,999983115 & 0,0218417 \\
5 & $-0,2912320593$ & 0,999970877 & 0,0217903 \\
6 & $-0,4456811305$ & 0,999955432 & 0,0217115 \\
7 & $-0,6321977548$ & 0,99993678 & 0,0215998 \\
8 & $-0,8507819321$ & 0,999914922 & 0,0214495 \\
9 & $-1,1014336626$ & 0,999889857 & 0,0212549 \\
10 & $-1,3841529462$ & 0,999861585 & 0,0210103 \\
11 & $-1,6989397829$ & 0,999830106 & 0,0207103 \\
12 & $-2,0457941728$ & 0,999795421 & 0,0203490 \\
13 & $-2,4247161157$ & 0,999757528 & 0,0199208 \\
\hline
\end{tabular}

In table 3. The value of $\left|M_{\text {exp }}-M_{t h}\right|$ at $k=12$ is $0,0203 u$, which is smaller than the result getting by SEMF before modification $\left(\left|M_{\text {exp }}-M_{t h}\right| \approx\right.$ $0,0204 u$ ). This value indicates that the mass calculation using Modified SEMF is better than before modification that it gives the value closes to the experimental results $\left(M_{\text {exp }}\right)$. Moreover, it will be more accurate if calculate it using computation.

Next step, for more convincing, the results in table 3 were plotted (figure 2). If we focused on nuclei $\mathrm{Pb}_{82}^{208}$, with atomic mass $A=208$, the value of $\frac{E}{E_{0}}=$ $\left(1+b_{k} \hat{\mathrm{a}}_{k}^{2}\right)=0,999$ at $\hat{\mathrm{a}}_{2}$ and $\hat{\mathrm{a}}_{4}$ equal to 0,01 corresponding with the curve figure 2.a. Moreover, for the figure 2.b show the curve of $b_{k}$ function of mass number $A$, written by $b_{k}(A)$, for nuclei $\mathrm{Pb}_{82}^{208}$, with atomic mass $A=208$, the value of $b_{2}=-0,02, b_{3}=$ $-0,08, b_{4}=-0,17$, and $b_{6}=-0,44$.
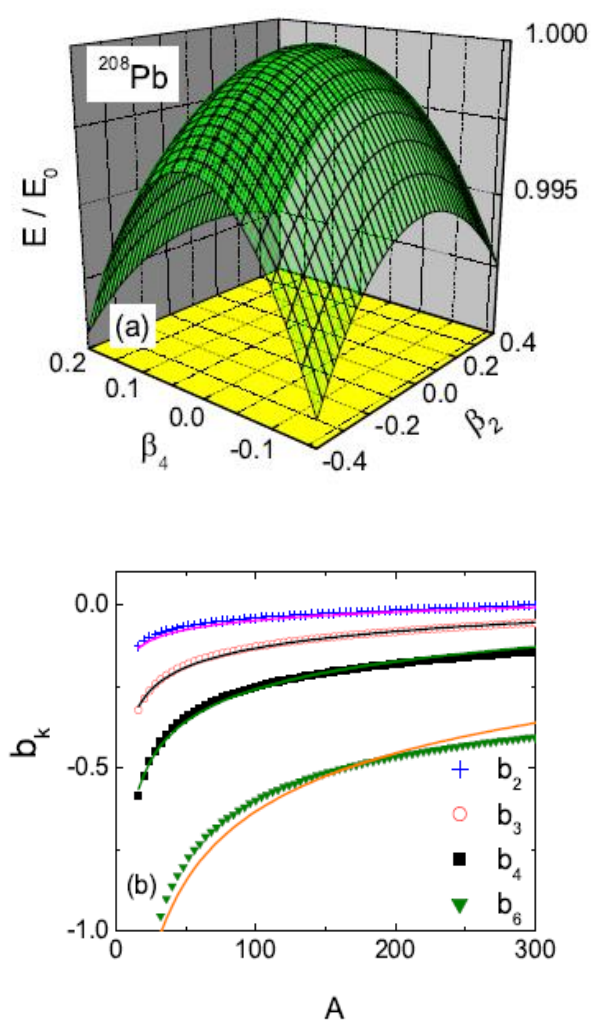

Figure 2. (a) Energy of $\mathrm{Pb}_{82}^{208}$ with respect to $\hat{a}_{2}$ and $\hat{a}_{4}$ deformation with skyrme density approach. (b) The value of $b_{k}$ as a function of mass number. The scattered symbols denote the obtained curvatures of the parabolas with the skyrme force SKM* for a number of nuclei. The solid curves denote the corresponding results with an empirical formula (Wang $\mathrm{N}$ et al., 2010).

From the explanations above, we can conclude that modified Semi-Empirical Mass Formula (SEMF) is obtained better result on calculation the mass of nuclei than SEMF before modification, it is also proved the correspondency of theoretical results with experimental results were showed in figure 2 .

Second,by comparing Modified SEMF of Liquid Drop Model (LDM) with other models such as Finite Range Droplet Model (FRDM), Hartree Fock Bougolibouv (HFB-14, and HFB-17) using accuracy parameters in the form of rms deviation (ó(M)) and number of model parameters $\left(\mathrm{N}_{\mathrm{p}}\right)$ is given in table 4 .

Table 4. The value of rms deviation ó(M)refers to 2149 measured 
masses and number of model parameters $\left(\mathrm{N}_{\mathrm{p}}\right)$ are used in each models (Wang $\mathrm{N}$ et al., 2010).

\begin{tabular}{lllll}
\hline Parameters & FRDM & $\begin{array}{l}\text { HFB- } \\
\mathbf{1 4}\end{array}$ & $\begin{array}{l}\text { HFB- } \\
\mathbf{1 7}\end{array}$ & $\begin{array}{l}\text { LDM (Modified } \\
\text { SEMF) }\end{array}$ \\
\hline ó $(M)$ & 0,656 & 0,729 & 0,581 & 0,516 \\
$N_{p}$ & 31 & 24 & 24 & 15 \\
\hline
\end{tabular}

The corresponding rms deviation of nuclear masses for the 2149 measured nuclei with the parameter set LDM (Modified SEMF) is listed in table 4 then the result from other models (FRDM, HFB-14, and HFB17) are also listed for comparison. Compared with the FRDM, the rms deviation is dreadfully reduced $21 \%$ from $0,656 \mathrm{Mev}$ to $0,516 \mathrm{Mev}$. And if it compared with HFB-14 and HFB-17 which have rms deviation (ó(M)) 0,729 Mev and 0,581 Mev, respectfully. LDM still remain smaller than them. It indicates LDM is the best model to calculate the mass of nuclei. In addition, compared using number of model parameters $\left(\mathrm{N}_{\mathrm{p}}\right)$ used in each models. The number of parameters in the models is reduced from 31 to 15 consists of 13 macroscopic model parameters $\left(a_{v}, a_{s}, a_{c}, c_{s y m}\right.$, $\left.\mathcal{K}, a_{\text {pair }}, g_{1}, g_{2}, c_{1}, V_{0}, r_{0}, a, \ddot{e}_{0}\right)$ and two microscopic model parameters (ã and $p$ ) (Wang $\mathrm{N}$ et al., 2010). The value of model parameters was obtained by fitting to experimental results, as a reason it is called semiempiric.

\section{Discussion}

This paper proposed to explain how the isospin effects should take into account of Modified Semi-Empirical Mass Formula (SEMF) in Liquid Drop Model (LDM) by equation $I=\frac{(N-Z)}{A}$, Modified SEMF tuns to be more accurate than before modification .In addition, two accuracy testing were applied, by comparing $\mid \mathrm{M}_{\text {exp }}-$ $\mathrm{M}_{\mathrm{th}} \mid$ for nuclei $\mathrm{Pb}_{82}^{208}$ using SEMF before and after being modified and by comparing Modified SEMF with other models such as FRDM, HFB-14, and HFB17 using accuracy parameters in the form of rms deviation (ó(M)) and number of model parameters $\left(\mathrm{N}_{\mathrm{p}}\right)$. We have shown that Modified SEMF is the most accurate formulation to calculate nuclear binding energy and mass of the nuclei indicates with small value of rms deviation (ó(M)) and number of model parameters $\left(\mathrm{N}_{\mathrm{p}}\right)$.

\section{CONCLUSION}

Based on literature (Wang $\mathrm{N}$ et al., 2010) the value of $\left|\mathrm{M}_{\exp }-\mathrm{M}_{\mathrm{th}}\right|$ for nuclei $\mathrm{Pb}_{82}^{208}$ using SEMF before modification is $0,0204 u$ and for modified SEMF we obtained $\left|\mathrm{M}_{\exp }-\mathrm{M}_{\mathrm{th}}\right| \approx 0,0203 u$ at $k=12$. The value of $\left|M_{\text {exp }}-M_{t h}\right|$ for modified SEMF is smaller than before modification, it indicates that Modified SEMF is a good formula to calculate the mass of nuclei.Furthermore, by comparing Modified SEMF with other models such as FRDM, HFB-14, and HFB17 using accuracy parameter in the form of rms deviation (ó(M)) and number of model parameters $\left(\mathrm{N}_{\mathrm{p}}\right)$. Based on the research that has been done shows that rms deviation (ó(M)) decrease $21 \%$ to 0,516 and number of model parameters $\left(\mathrm{N}_{\mathrm{p}}\right)$ decrease to 15 , consists of 13 macroscopic model parameters $\left(a_{v}, a_{s}, a_{c}, c_{\text {sym }}, \mathcal{K}, a_{\text {pair }}, g_{1}, g_{2}, c_{1}, V_{0}, r_{0}, a, \ddot{e}_{0}\right) \quad$ and two microscopic model parameters (ã and $p$ ).

\section{ACKNOWLEDGEMENTS}

We thank to Dra. Eko Tri Sulistyani, M.Sc for a teamwork and careful reading of the manuscript. We also thank to all friends for all valuable suggestions and supports , This paper was supported by Research Group of Astrophysics, Cosmology and Mathematical Physics, Physics Department, Gadjah Mada University.

\section{REFERENCES}

Bartel J, Quentin P, Brack M, Guet C, Hakansson HB. 1982. Towards a better parametrisation of skyrme-like effective forces: a critical study of the skm force. Nuclear Physics 386: 79-100.

Greiner W, Müller B. 1989. Quantum Mechanics-Symmetries. Springer-Verlag, Berlin Heidelberg.

Griffith D. 2008. Introduction to Elementary Particles. second edition. WILLEY-VCH, Weinheim.

Meyerhof WE. 1967. Elements of Nuclear Physics. Stanford University, McGRAW-HILL Book Company, New York.

Wang N, Liu M, Wu X. 2010. Modification of nuclear mass formula by considering isospin effects, Physical Review C - Nuclear Physics 81: 1-19.

Wang M, Audi G, Kondev F.G, Xu X, Pfeiffer B. 2012. Atomic mass evaluation; AIP Conference Proceedings. 1484: 79-84. 